\title{
Traffic Incident Detection Based on the Grid Model
}

\author{
Wei-Lieh Hsu 1,*, Po-Lun Chang², Rueiher Tsaur ${ }^{1}$ \\ ${ }^{1}$ Department of Computer Information and Network Engineering, Lunghwa University of Science and Technology,33306, Taoyuan, \\ Taiwan \\ ${ }^{2}$ Department of Electrical Engineering, Lunghwa University of Science and Technology,33306, Taoyuan, Taiwan \\ *Corresponding Author: Leih@ms2.hinet.net
}

Copyright (C) 2013 Horizon Research Publishing All rights reserved.

\begin{abstract}
Highway accidents significantly impact normal traffic flow. Consequently, automatic detection of abnormal traffic events has gradually attracted the attention of researchers interested in intelligent transportation system. This work presents a vision-based approach for automatic traffic congestion and incident detection. The proposed approach involves extracting entropy-based features to create a grid model that simulates dynamic traffic flow behavior. When an unusual event occurs in the lane of the vehicle employing the system, the system can immediately detect it and issue signals to approaching vehicles to prevent accidents. Experiments conducted using various simulation results clearly demonstrate the validity and effectiveness of the proposed approach for managing traffic congestion and detecting incidents.
\end{abstract}

Keywords Intelligent Transportation System, Entropy-based, Grid model, Traffic Congestion

\section{Introduction}

Several investigations have suggested that most highway accidents result from primary traffic incidents. Evidence demonstrates that over $50 \%$ of traffic accidents occur within 10 minutes following primary incidents from Versavel [1]. Thus it is helpful to devise an automatic traffic event detection system to promptly detect abnormal events and thus reduce the occurrence of traffic accidents.

Numerous recent studies had examined the problem of incident detection by Caliendo et al. [2] and Park et al. [3]. Ozbay et al [4] defined traffic incidents as unusual events that block a lane, including stopped vehicles, fallen objects, and chemical spills. Research on incident detection has frequently stressed identifying incidents from historic data. Wang proposed a method based on the fast Mallat algorithm of wavelet analysis for detecting traffic incidents, and simulation results demonstrate that this method performs well [5]. However, the complexity of those approaches made it unsuitable for real-time applications. Furthermore, Kamijo proposed an accident recognition system based on the Hidden Markov Model (HMM) [6]. This system can learn various event patterns and identify current events. However, the system function is limited to identifying collisions and traffic jams in intersections. Binglei employed a fuzzy-logic based algorithm to detect highway traffic incidents, and adopted the membership function and fuzzy rules to detect and assess traffic flow status. Simulation results indicated that the proposed method had low false alarm and high detection rate under uniform traffic volume [7]. However, traffic volume usually changes with time. Jin presented a novel method for freeway incident detection based on a constructive probabilistic neural network [8]. However, since the scene of an accident is typically messy, it is difficult to obtain useful event patterns from learning processes with image-based approaches. According to the application of pattern classification, Xiaorong proposed a Fuzzy Learning Classifier System in automatic traffic incident detection, the field traffic incident database I-880 is adopted to build an evaluation environment. Compare with Multi Forward Neural Network with Back-Propagation Algorithm, the results show the proposed system (FLCS) has lower false detection rate and shorter mean detection time [9]. Kamino proposed a traffic monitoring system based on the ST-MRF model for detecting slow vehicles, traffic congestion and incidents [10]. This system detects and assesses abnormal traffic events using a semantic hierarchy algorithm containing three classes: coordinate-class, behavior-class and event-class, which are designed to be robust against occlusion and illumination changes. The system was applied at two different locations over several months and proved highly effective with few false alarms owing to illumination changes and heavy traffic. However, the vision-based approach for incident detection has advantages over traditional loop data techniques in terms of flexibility, and ease of installation and maintenance.

The fact that vehicles have various colors, sizes and models hinders real-time event detection and identification using image-based approaches. This study devises entropy-based features to construct a grid model, and defines a set of local rules to resolve the problem. If certain unusual events violate local rules, they are considered 
abnormal events and further checks are performed to determine whether they represent traffic congestion. The proposed method is highly suitable for practical applications due to its efficiency. Therefore, the system can immediately forward the variable message sign of abnormal events to drivers or traffic billboards, and informed drivers can either determine optimal routes to their destinations or decrease their speed to prevent accidents.

The remainder of this paper is organized as follows. Section 2 presents an overview of entropy. Section 3 then presents the proposed method based on the grid model for real-time traffic event detection, after which Section 4 presents experimental results. Finally, Section 5 presents conclusions.

\section{Overview of Entropy}

This section briefly addresses the theoretical basis of the proposed method, particularly its entropy-based characteristic. Entropy is a parameter commonly used to rate system disorder in thermodynamics. Shannon's entropy is a special form of entropy commonly used in information theory, particularly signal processing. This investigation adopts exponential entropy, defined by Pal et. al. [11] as the measurement for useful feature extraction, and exponential entropy is defined as follows:

$$
H=E(I)=\sum_{i=1}^{n} p_{i} \exp \left(1-p_{i}\right)
$$

Equation (1) shows that maximum entropy is reached when all constituent probabilities are equal. Additionally, exponential entropy is uniformly distributed and has a distinct upper bound. Restated, if

$$
\begin{aligned}
p_{1}=p_{2}= & \ldots=p_{n}=\frac{1}{n}, \text { then } \\
& \lim _{n \rightarrow \infty} \sum_{i=1}^{n} p_{i} \exp \left(1-p_{i}\right)=\lim _{n \rightarrow \infty} \exp \left(1-\frac{1}{n}\right)=e .
\end{aligned}
$$

Clearly, the value of the exponential entropy approaches a constant when the probabilities of occurrence are uniformly distributed and value of $\mathrm{n}$ approaches infinity. In comparison, the value of Shannon's entropy does not stabilize but continues to grow under the same condition. Exponential entropy has another important property, namely that if the detection zone contains more blocks with connected components, then its corresponding entropy value is large, as illustrated in section 3.1. Therefore, the exponential entropy better fits the vision-based traffic monitoring system.

\section{Proposed Method}

Traffic surveillance cameras are usually mounted on raised locations beside a road to facilitate monitoring of traffic in all lanes. To detect abnormal events, this study employs background differencing to obtain the difference between the current and the reference background images, $I$ and $B$. The difference is then digitized to obtain a foreground object map, as shown in Fig. 1.

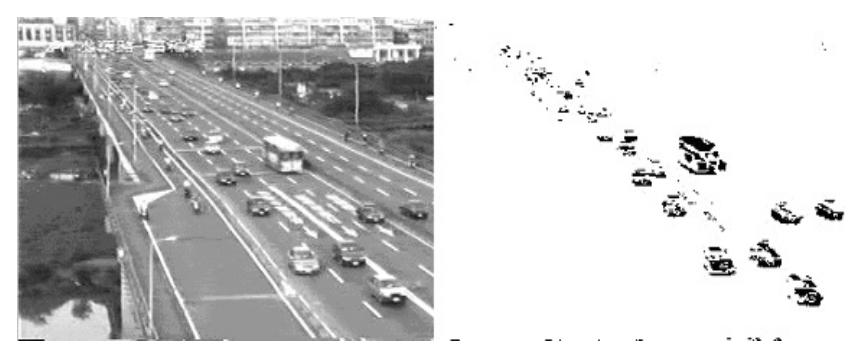

Figure 1. Left: The original image. Right: The corresponding foreground object map of the measured area.

The equation for the subtraction process is as follows:

$$
M(p)=\left\{\begin{array}{lll}
1 & \text { if } & \left|I_{k}(p)-B(p)\right|>I_{t h} ; \\
0 & \text { if } & \left|I_{k}(p)-B(p)\right| \leq I_{t h},
\end{array}\right.
$$

where $I_{k}(p)$ and $B(p)$ denote the current and background reference images at pixel $p$ of frames $k$ and $B$, respectively. Moreover, $M$ represents a binary image, and $I_{\text {th }}$ denotes a predefined threshold capable of recognizing the exponential entropy change between the two consecutive frames.

\subsection{Grid Model}

To efficiently detect abnormal events, the active pixels can be used as an underlying feature for moving vehicles following background differencing. The measured area contains numerous lanes, each of which is divided into several unit areas. The length of each unit area is taken as the average length of a typical car, as shown in Fig. 2.

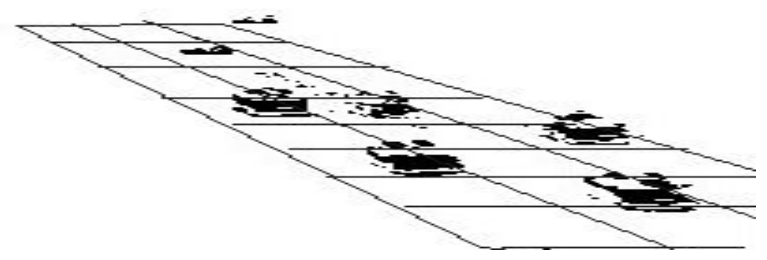

Figure 2. A binary image after the background differencing, and each lane of measured area is divided into several unit areas.

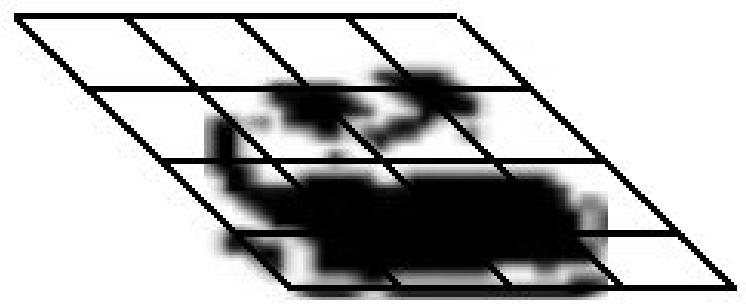

Figure 3. The distribution of active pixels in a unit area.

Next, each unit area is divided into $\mathrm{n}$ blocks, as shown in Fig. 3, where the probability $p_{i j}$ of each block can be 
calculated as follows.

$$
P_{i j}=\frac{N_{i j}}{N_{j}}, \quad \text { where } \quad N_{j}=\sum_{i=1}^{n} N_{i j} .
$$

$N_{i j}$ denotes the number of active pixels in the $j$ th block of the ith unit area, and $\mathrm{Nj}$ represents the total number of active pixels in the ith unit area. Under these circumstances, the exponential entropy of the unit area can easily be calculated using Eqs.(4) and (1).

The exponential entropy has important properties for real-time applications in intelligent transportation system. Namely, the corresponding entropy is larger if the detection zone contains more blocks with connected components [12]. The proof is briefly illustrated below. Consider two different unit areas, and let the total number of blocks in each detection zone be $\mathrm{n}$. Assume that $n^{\prime}$ blocks containing connected components exist in unit area 1 , while unit area 2 contains $n^{\prime \prime}$ blocks with connected components. If $n^{\prime}<n^{\prime \prime}<n$, then let the probability distributions of unit area 1 and unit area 2 be $P^{\prime}=\left(p^{\prime}(1), p^{\prime}(2), p^{\prime}(3), \ldots, p^{\prime}\left(n^{\prime}\right), \ldots, p^{\prime}(n)\right) \quad$ and $P^{\prime \prime}=\left(p^{\prime \prime}(1), p^{\prime \prime}(2), p^{\prime \prime}(3), \ldots p^{\prime \prime}\left(n^{\prime \prime}\right), \ldots, p^{\prime \prime}(n)\right)$, respectively. In the $P^{\prime}$ sequence, the elements before $p^{\prime}\left(n^{\prime}+1\right)$ are all non-zeros and have no order, while all other elements are zero. In the case of the $P^{\prime \prime}$ sequence, the components preceding $p^{\prime \prime}\left(n^{\prime \prime}+1\right)$ are non-zeros and non-ordered. Without loss of generality, this study assumes the case where the $P^{\prime \prime}$ sequence contains a single large $p^{\prime \prime}(i)$ and several tiny $p^{\prime \prime}(i)$ do not exist, since the tiny $p^{\prime \prime}(i)$ can be considered to be noise. According to the Schur-Concavity property [13], non-zero components can be picked up without repetition from $P^{\prime}$ and $P^{\prime \prime}$ to satisfy

$$
\sum_{i=1}^{k} p^{\prime \prime}(i) \leq \sum_{i=1}^{k} p^{\prime}(i)=1, \text { for } k=1,2, \ldots, n .
$$

Here $P^{\prime \prime}$ is majorized by $P^{\prime}$, represented as $P^{\prime \prime} \prec P^{\prime}$ [13]. Based on the Schur-Concavity property, the exponential entropy $H$ is also Schur-concave, implying that

$$
H\left(P^{\prime}\right)<H\left(P^{\prime \prime}\right) \text {. }
$$

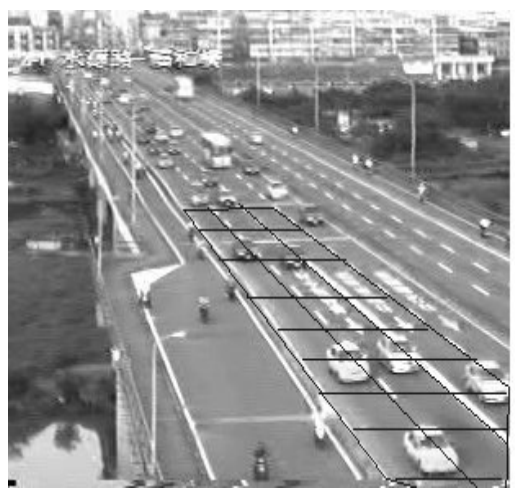

Thus a detection zone with more blocks containing connected components will have high corresponding entropy even if some blocks have low probabilities. Owing to possessing this proper characteristic, the entropy measurement is well qualified for use in detecting moving vehicles.

Each unit area can be considered a unit component in the grid model [12]. Since vehicles generally follow a certain direction, the status value is represented by the total exponential entropy change in its corresponding unit area, which can be obtained as follows:

$$
S_{i, j}(n)=\left\{\begin{array}{clcc}
S_{i, j}(n-1)+1 & \text { if }\left|H_{i, j}(n)-H_{i, j}(n-1)\right|>H_{t h} & \text { and } & H_{i, j}(n) \neq 0 \\
S_{i, j}(n-1) & \text { if }\left|H_{i, j}(n)-H_{i, j}(n-1)\right| \leq H_{t h} & \text { and } & H_{i, j}(n) \neq 0 \\
0 & \text { if } & \text {; }
\end{array} \quad\right. \text {; }
$$

where $H_{i, j}(n)$ and $H_{i, j}(n-1)$ represent the exponential entropy value of the unit area located at the ith unit area in the jth lane for the current and previous frames, respectively, and $S_{i, j}(n)$ and $S_{i, j}(n-1)$ are the corresponding status values; Moreover, $H_{t h}$ represents a pre-determined light threshold. Therefore, the proposed model comprises a number of regular grids with a simple identical grid, and the state can be one among a finite $k$ possible numbers. A larger status value indicates that the unit area is occupied by a slow moving vehicle. A constantly invariant status value indicates that a stopped vehicle is located on the area. Highway traffic can be represented as a dynamic discrete system involving discrete space, time and finite state. The following equation describes the dynamic discrete system.

$$
\mathrm{Z}\left(n^{\prime}\right)=\left[\begin{array}{ccccc}
S_{1,1} & S_{1,2} & . . & . . & S_{1, m} \\
S_{2,1} & & & & S_{2, m} \\
: & & & & : \\
S_{n, 1} & S_{n, 2} & . . & . . & S_{n, m}
\end{array}\right],
$$

where $S_{i, j}$ denotes a grid value, $n^{\prime}$ represents the frame number, $m$ is the number of lanes, and $n$ represents the number of unit area in a lane. Figure 4 illustrates the proposed model and its corresponding varying cellular states.

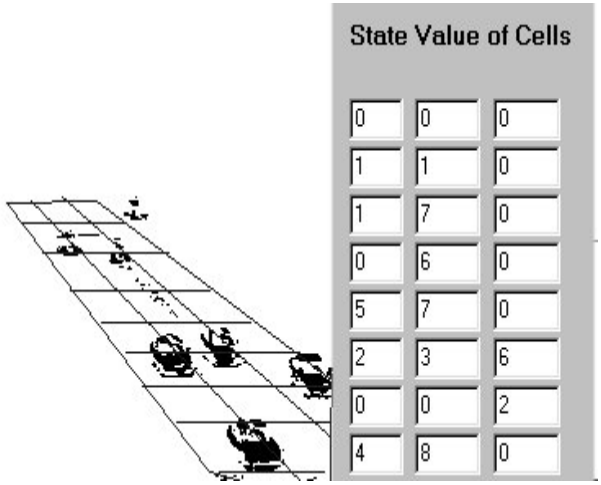

Figure 4. The proposed model and its corresponding varying cellular states. 


\begin{tabular}{|c|c|c|c|}
\hline$(i-1, j-1)$ & $(i, j-1)$ & $(i+1, j-1)$ & \\
\hline$(i-1, j)$ & $(i, j)$ & $(i+1, j)$ & The Moving \\
\hline$(i-1, j+1)$ & $(i, j+1)$ & $(i+1, j+1)$ & \\
\hline
\end{tabular}

Figure 5. The relative cell locations in the proposed model.

\subsection{Event Detection}

To detect abnormal events more efficiently, this study defines a local rule to manage the dynamic behavior of traffic flow. If certain traffic events occur and violate the local rule, the system responds to the situation as an unusual event and further checks to determine whether a traffic incident has occurred. Figure 5 shows the relative cell locations proposed in the proposed model, and the rules used to manage these cells are presented below.

$$
\begin{array}{lllll}
\text { Rule } & 1 . & \text { If } & H_{i, j}(n)=0 \quad, \quad \text { then } \quad S_{i, j}(n)=0 . \\
\text { Rule } & 2 . & \text { If } & H_{i, j}(n) \neq 0 \quad, \quad \text { then } \quad S_{i, j}(n)=S_{i, j}(n-1)+1 . \\
\text { Rule } & 3 . & \text { If } & H_{i, j}(n) \neq 0 \quad \text { and } \quad S_{i, j}(n-1)=S_{i, j+1}(n-1)=0, \\
& & \text { then } & S_{i, j}(n)=S_{i-1, j+1}(n-1) \quad \text { or } \quad S_{i+1, j+1}(n-1) .
\end{array}
$$

where zero state value represents the False Boolean value, and non-zero state value represents the True Boolean value. Rule 1 indicates that if no vehicle occupies the unit area, its corresponding exponential entropy value should be zero. In this circumstance, it's the state value is set to zero. Rule 2 means that if the state of a cell is non-zero, then a vehicle is occupying the area. Since a vehicle is always moving in a certain direction, the cellular state value is increased by one after every frame, with a slower vehicle corresponding to a larger state value. Rule 3 is designed to describe the case of a vehicle making a lane change. Rule 3 means that when a cell and its following cell have a zero state in the previous frame, no vehicles exist in the area. The state value may become one when if a neighboring vehicle does a lane change. Events that violate the local rule are considered abnormal. For example, if the state value remains invariant, that is $S_{i, j}(n+1)=S_{i, j}(n)$, then the unit area is occupied by stopped vehicles. Normally, a vehicle that randomly stops on a freeway is considered illegal and very dangerous, and thus definitely constitutes an abnormal event. To represent an abnormal event effectively, this study uses a status matrix to represent the occurrence of an incident. In situations involving an inactive cell, the value of its corresponding element in the status matrix $b_{i j}$ is activated and is set to 1 . On the other hand, an active cell corresponding to a status matrix element is designated as 0 . An $\mathrm{n} * \mathrm{~m}$ status matrix can be represented as follows.

$$
M_{s}=\left[\begin{array}{ccccc}
b_{1,1} & b_{1,2} & . . & . . & b_{1, m} \\
b_{2,1} & & & & b_{2, m} \\
: & & & & : \\
b_{n, 1} & b_{n, 2} & . . & . . & b_{n, m}
\end{array}\right],
$$

where $b_{i j}$ denotes a binary value, $\mathrm{m}$ represents the number of road lanes and $\mathrm{n}$ is the number of unit areas for each lane. A column of the status matrix represents the traffic situation of each lane. Columns containing any nonzero element indicate that stopped vehicles occupying the corresponding lane.

Usually, a detection zone covers numerous lanes, each of which is divided into several unit areas via two state values. Thus, the total possible states of a measured area are very large. A real-time quota is necessary to analyze each case. Thus this study employs the Tanimoto measure [14] to calculate the similarity and distinguish partial road blockages from traffic congestion, as follows.

$$
d_{T}(X, Y)= \begin{cases}\frac{X Y^{\prime}}{X X^{\prime}+Y Y^{\prime}-X Y^{\prime}} & \text { if } X \text { or } Y \neq 0 \\ 0 & \text { if } X \text { and } Y=0 .\end{cases}
$$

The Tanimoto measure indicates the similarity of common attributes possessed by binary vectors $\mathrm{X}$ and $\mathrm{Y}$, where $\mathrm{X}$ and $\mathrm{Y}$ are the vectors that contain the column elements of the status matrix Ms. The Tanimoto measure represents the traffic situation of a specific lane. Meanwhile, $Y^{\prime}$ is the transpose of $Y$ vector. When car speed in vehicular lane slows significantly, there is a potential danger to approaching vehicles. The proposed algorithm creates nonzero elements in the state matrix and indicates an abnormal event in the unit area.

Next, this study defines the $D_{T}$ measure, which is used to assess whether the measured area is only partially blocked or all lanes are blocked thoroughly. The definition is given below:

$$
D_{T}=\operatorname{Min}\left(d_{T}\left(L_{1}, L_{2}\right), d_{T}\left(L_{2}, L_{3}\right), \ldots d_{T}\left(L_{n-1}, L_{n}\right)\right)
$$

where $L_{i}=\left(b_{i 1}, b_{i 2}, \ldots, b_{i m}\right)$ denotes the column elements of the status matrix, which represent the traffic status for lane $i$. In the case of dependent multilane, vehicles usually do not travel in a specific lane, thus the traffic situation of all lanes should be similar. If $D_{T}$ equals zero, which means some nonzero elements exist in the status matrix and the traffic situation differs between lanes, a lane may be affected by an accident, unexpected object stops, or construction activity, approaching drivers are informed to decrease speed for safety reasons. Nonzero value of $D_{T}$ implies traffic congestion. Approaching drivers thus should be prompted to decrease speed or if possible take an alternative route.

Finally, the algorithm of the proposed method is rechecked when the values of the elements in the status matrix are changed. The approach is summarized as follows.

Algorithm for abnormal traffic event detection:

Step1. If the values of the status matrix elements are zero.

$$
\left(\sum_{i} \sum_{j} b_{i j}=0\right)
$$


Traffic keeps moving.

Step2. If nonzero elements exist in the status matrix.

$$
\left(\sum_{i} \sum_{j} b_{i j} \neq 0\right)
$$

Abnormal traffic events are detected.

If $D_{T}=0$

Partial blocking exists that may be caused by accident or illegal parking.

Else $D_{T} \neq 0$

Traffic congestion exists.

\section{Experimental Results}

The experiments use the video sequences of highway traffic provided by the Bureau of Transportation in Taiwan. The sequences are taken using a rate of 10 frames per second, and the width and height of each image frame are 320 and 240 pixels, respectively. The measured area contains three lanes and each lane is divided into several unit areas, where the length of each unit area is taken as the average length of a typical car. Figure 6 shows the system interface at a particular instant. This study conducted several simulations involving relatively uniform traffic, such as freeways, and found the model to have good performance under the sunny weather conditions, and the results are shown in terms 1 and 2 of Table 1 . The approach is also applied to complex traffic in urban environments, where cars, motorcycles and bicycles all share the same lanes, and accuracy is consistently high. When traffic flow is heavy, the accuracy rate clearly reduces due to the entropy value being affected by moving vehicles and rainy weather conditions. The experimental results for a vehicle illegally stopped in the left lane are shown in Figs. 7 and 8. Figure 7 shows the blocked unit areas, while Fig. 8 illustrates the variation of the unit areas occupied by a stopped truck, and as well as the time at which the blocking begins. This curve can be used to estimate that the truck had stopped in the left lane for about 1 minute, with the curve value reaching zero at the time when the truck left. Additionally, the measure $D_{T}=\mathbf{O}$ shown in Fig. 7 indicates an unusual event but that not all road lanes are congested.

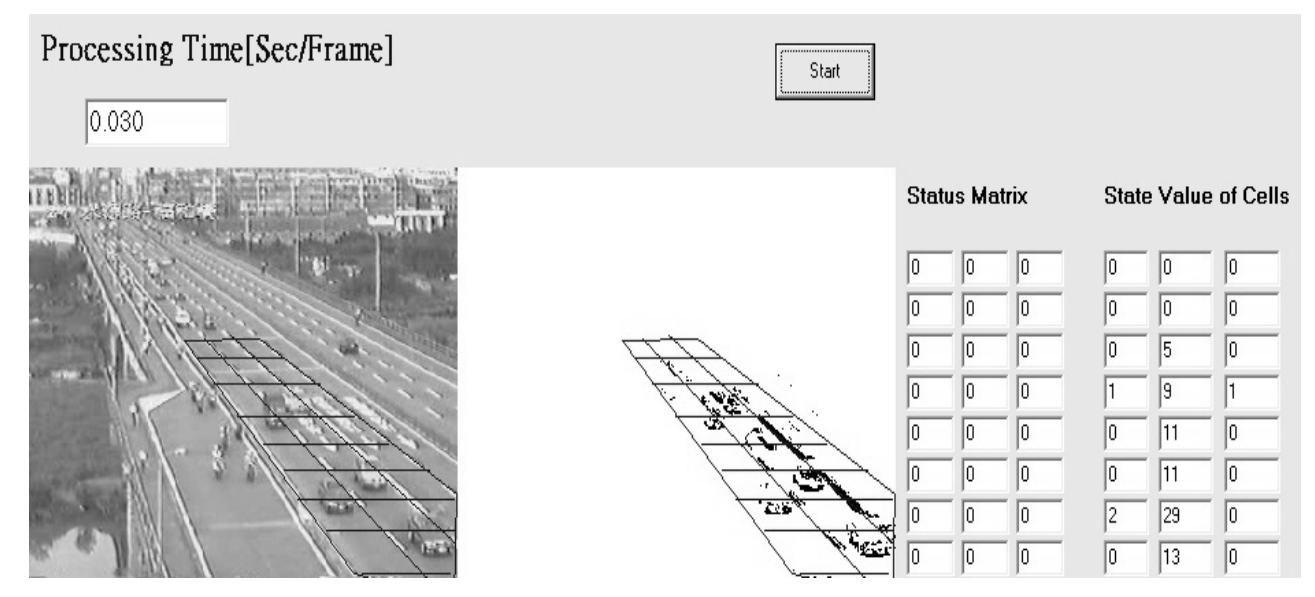

Figure 6. The system interface at a particular instant.
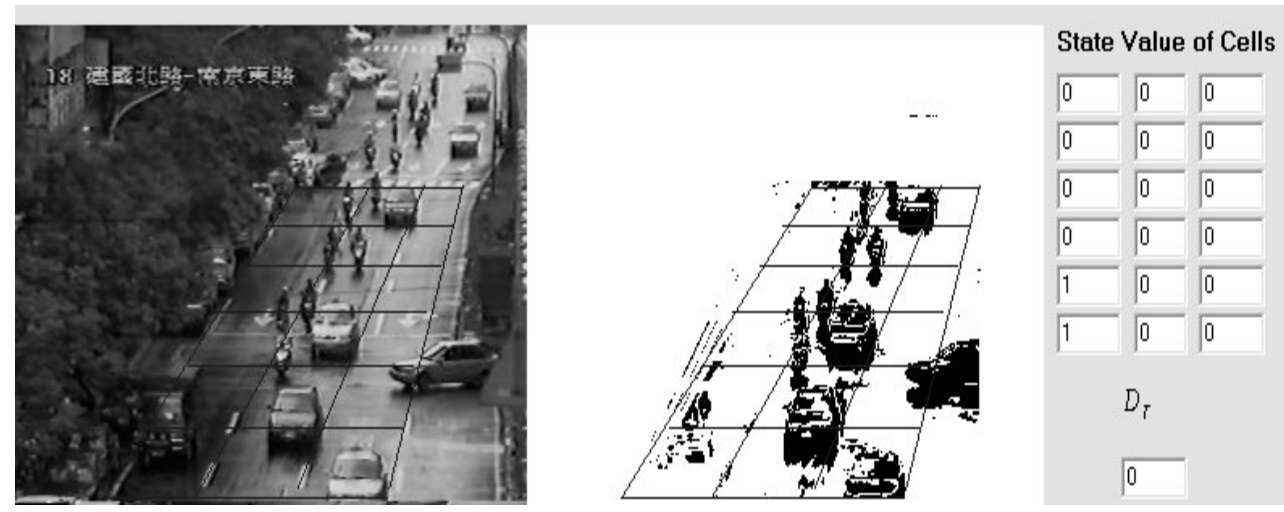

Figure 7. The original image and its binary image, and its variation of status value for a consecutive video, which indicates a vehicle illegally stopped on the left lane. 


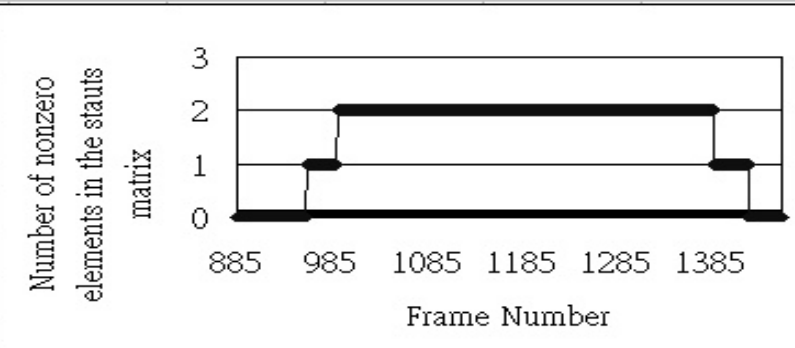

Figure 8. The corresponding measure for the result of Figure 7. The variation of nonzero elements in the status matrix..

This study also performed several experiments involving vehicles stopped at an intersection waiting for a red light; Fig. 9 shows the results. Figure 9 illustrates the variation of blocked unit areas at an intersection, and the $D_{T}$ measure is 1 , indicating all the lanes are blocked by the stopped vehicles.



Figure 9. The original image and its binary image, and the variation of cellular states calculated for a consecutive video, which indicates all lanes are blocked by stopped vehicles.

Table 1 illustrates the detection rate under different traffic situations. Two possible cases exist for the error image frame: false positive and false negative. False positive means the status matrix contains the nonzero element when the traffic is still moving in its corresponding unit area. Meanwhile, false negative means the unit area is blocked, but its corresponding element value is still zero, and occurs when the color of the vehicle approaches that of the background. From the listed results, the proposed approach clearly can effectively detect incidents. Moreover, the accuracy rate is very high and independent of the time duration. However, items 3 and 5 fail when two vehicles move very closely together and simultaneously many motorcycles are present, which affect the entropy value, causing the system to falsely detect a stopped vehicle. The results shown in term 4 were obtained during heavy traffic, causing cars to move very slowly, and slightly reducing the entropy variation in the corresponding unit areas. The proposed system falsely detected stopped vehicles under this condition, negatively impacting the accuracy rate. On the other hand, the $D T$ measure cannot perform well when applied to a single lane, such as a freeway off-ramp.

All tests are implemented on a Duo CPU, 2.0 GHz PC. The proposed system can process each image frame at a rate of 0.03 seconds/frame, a sufficiently economic computational time, making it more appropriate than existing alternatives for real-time applications. Additionally, the approach based on the entropy-based feature is insensitive to partial changes in lighting conditions. However, shadow and moving motorcycles significantly impact the entropy computation and detection rate. Therefore, this traffic incident detection approach is more suitable for use on highways where motorcycles are prohibited.

Ly et al. [15] indicated traffic incident is an unusual event that reduces the capacity of road, like traffic accident, stopping vehicles and so on, that significantly affects the service quality of road. Ozbay et al. [4] defined traffic incident as an abnormal event that caused blockage of traffic lane. Then based on these concepts, it verify the correctness and accuracy of the proposed method, since stopping vehicles are detected on traffic lane at any times, that is very dangerous and reduces the traffic capacity of road obviously.

Recently, many different type of neural network [16], such as PNN, MLF, CPNN etc, are adopted to address the automatic incident detection, their experimental results show the desirable performance with test data, these approaches usually regarded as one of the most promising method. However, an appropriate configuration of neural networks is determined for each application by performing a lot of experiments, that is time-consuming, and the optimal parameter setting is chosen by training with historic data. Normally, there are not historical data of traffic incidents for every interested lane; while our proposed method can recognize traffic incident in a short duration without priority knowledge, which it is more appropriate to real- time application, so the efficiency is better than the method of neural network.

Table 1. The experimental results under different traffic events.

\begin{tabular}{|c|c|c|c|c|c|}
\hline \multirow{2}{*}{ Item } & \multirow{2}{*}{ Unusual events } & \multirow{2}{*}{ Length of sequence (frames) } & \multicolumn{2}{|c|}{ Number of error image frames (frames) } & \multirow{2}{*}{ Detected Rate (\%) } \\
\cline { 4 - 6 } & & False positive & False negative & \\
\hline 1 & Normal & 380 & 0 & 0 & 100 \\
\hline 2 & Accident & 500 & 0 & 0 & 100 \\
\hline 3 & Illegal parking & 1430 & 31 & 0 & 97.8 \\
\hline 4 & Illegal parking & 520 & 70 & 10 & 84.6 \\
\hline 5 & Congestion & 450 & 20 & 0 & 95.5 \\
\hline
\end{tabular}




\section{Conclusions}

Traffic accidents are a growing global problem, with escalating social costs. Thus the availability of innovative accident prevention facilities installed along major highways, such as automatic traffic event detection devices, is increasingly important. This study uses entropy-based features to create a cellular model involving discrete space, time and finite state. This model can easily formulate the traffic behavior of multiple lanes using a time-varying $2 \mathrm{D}$ matrix. This study then defines a local rule for managing dynamic traffic flow. The system can promptly detect unusual events in a vehicular lane, and then warn approaching drivers to prevent accidents, significantly reducing accident rates. Additionally, the ease of system implementation makes it suitable for practical applications. This study has conducted multiple simulations, and the results demonstrate that the proposed system is both effective and efficient.

\section{REFERENCES}

[1] J. Versavel, "Road Safety through Video Detection." International Conference on Intelligent Transportation Systems, 753-757, 1999.

[2] C. Caliendo, M. Guida, and A. Parisi, "A Crash-prediction model for multilane roads," Accident Analysis and Prevention. Vol. 39, No. 4, 657-670, 2007.

[3] B. J. Park, and D. Lord, "Application of finite mixture models for vehicle crash data analysis," Accident Analysis and Prevention. Vol. 41, No.4, 683-691. 2009.

[4] K. Ozbay, and P. Kachroo, "Incident Management in Intelligent Transportation System,” Artech House, 1999.

[5] X. Wang and J. Zhang, "A traffic incident detection method based on wavelet algorithm," Proceedings of IEEE Workshop on Software Computing in Industrial Applications, pp. 166-172, 2005.

[6] A. Kamijo, Y. Matsushita, K. Ikeuchi, and M. Sakauchi "Traffic Monitoring and Accident Detection at Intersections,"
IEEE Transactions on Intelligent Transportation Systems, Vol. 1, No.2, 108-118, 2000.

[7] X. Binglei, H. Zehng and Hongwei, "M. Fuzzy-logic based traffic incident detection algorithm for freeway," Proceedings of the 7th International Conference on Machine Learning and Cybernetics, Kunming, 1254-1259., 2008.

[8] X. Jin, D. Srinivasan, and R. L. Cheu, "Classification of Freeway Traffic Patterns for Incident Detection Using Constructive Probabilistic Neural Networks," IEEE Transactions on Neural Networks. Vol.11, pp.1173-1187, 2001.

[9] S. Xiaorong, Z. Hai, F. Yaozu, and X. Chao, "Fuzzy Learning Classifier System and its Application Research in Automatic Traffic incident Detection," Proceedings of Industrial Electronics and Applications. Harbin, China. pp.769-772, 2007.

[10] S. Kamino, H. Koo, Xiaolu, Liu, K. Fujihira, And M. Sakauchi, "Development and Evaluation of Real-time Video Surveillance System on Highway based on Semantic Hierarchy and Decision Surface," IEEE International Conference on System, Man and Cybernetics, Vol. 1, $840-846,2005$.

[11] N. R. Pal, and S. K. Pal, "Entropy: A New Definition and its Applications," IEEE Transactions on System, Man and Cybernetics. Vol. 21, No. 5, 1260-1270, 1993.

[12] W. L. Hsu, H. Y. Mark, Liao, B. S. Jeng and K. C. Fan, "Real-time Traffic Parameter Extraction Using Entropy," IEE Proceedings of Vision, Image and Signal Processing. Vol. 151, 194-202, 2004.

[13] W. Marshall and I. Olkin, "Inequalities: Theory of majorization and its applications," CA: Academic Press, San Diego, 1979.

[14] J. T. Tou, and R. C. Gonzalez, Pattern Recognition Principles, Addison-Wesley, 1974.

[15] W. Ly, X. Liu and T. Zhu "A history data based traffic impact analyzing and predicting method," 2010 international conference on Electronics and information engineering, V2-219 -223. 2010.

[16] S. Chen, W. Wang, G. Qu and J. Lu, "Application of Neural Ensembles to Incident Detection", IEEE International Conference on Integration Technology, March 20-24, China, pp.388-393, 2007. 